\title{
Sense through wall human detection using UWB radar
}

Sukhvinder Singh" ${ }^{*}$, Qilian Liang ${ }^{1}$, Dechang Chen ${ }^{2}$ and Li Sheng ${ }^{3}$

\begin{abstract}
In this article, we discuss techniques for sense through wall human detection for different types of walls. We have focused on detection of stationary human target behind wall based on breathing movements. In detecting the breathing motion, a Doppler based method is used. Also a new approach based on short time Fourier transform is discussed and an already proposed clutter reduction technique based on singular value decomposition is applied to different measurements.
\end{abstract}

Keywords: UWB, Monostatic, Singular value decomposition, Short time Fourier transform, Discrete Fourier transform, Clutter reduction

\section{Introduction}

Detection of human target through wall is of interest for many applications. Military industry could use it for hostage rescue situations. In such scenarios, detection and location of humans inside a room is very critical as unknown building layout together with presence of armed persons can be dangerous for the rescuers. Another use could be for disaster search and rescue operations such as people trapped under building debris during earthquake, explosion or fire.

Ultra WideBand (UWB) technology has emerged as one of the preferred choices for such applications due to its good range resolution and good penetration through most of the building materials. High range resolution is a result of high bandwidth of UWB radar and it helps in better separation of multiple targets. Detection of human target is based on the fact that there is always some movement due to breathing or movement of body parts (as in case of a walking person). This small movement can be used to detect a human being from other objects behind a wall or beneath rubble but it becomes challenging due to high clutter from the wall and other objects inside a room.

\footnotetext{
* Correspondence: sukh84@gmail.com

'Department of Electrical Engineering, University of Texas at Arlington,

Arlington, TX, 76019-0016, USA

Full list of author information is available at the end of the article
}

The focus of this article is on detection techniques for a motionless human target using a monostatic UWB radar.

\section{UWB Overview and Features}

UWB systems are the ones which use signals with minimum $(10 \mathrm{~dB})$ bandwidth of $500 \mathrm{MHz}$ or fractional bandwidth of at least $20 \%$.

$$
\text { Fractional bandwidth }=2 \frac{f_{H}-f_{L}}{f_{H}+f_{L}}
$$

where, $f_{\mathrm{H}}$ and $f_{\mathrm{L}}$ are highest and lowest frequency points, respectively, with signal $10 \mathrm{~dB}$ below peak emission.

\section{A. Large bandwidth-high range resolution}

The relation between pulse width and radar range resolution is given as

$$
\text { Range resolution }=\frac{\tau \cdot c}{2}=\frac{c}{2 B}
$$

where $\tau=$ Pulse width in time domain, $B=$ bandwidth of the pulse, and

$c=$ speed of electromagnetic waves.

Good range resolution property of UWB can be used for localizing the target in an indoor environment.

\section{SpringerOpen ${ }^{\circ}$}

(C) 2011 Singh et al; licensee Springer. This is an Open Access article distributed under the terms of the Creative Commons Attribution License (http://creativecommons.org/licenses/by/2.0), which permits unrestricted use, distribution, and reproduction in any medium, provided the original work is properly cited. 


\section{B. UWB radar penetration through wall [1]}

As per the Electromagnetic theory, lower frequencies have better penetrating properties. UWB radar uses a large spectrum in combination with lower frequencies which makes it suitable for applications such as ground penetrating radar, foliage penetrating radar [2], and short-range radar to detect hidden objects behind walls. This penetration property is also of great importance for indoor location systems. Shorter wavelength makes possible use of smaller dimensions of receive and transmit antennas. On the other hand, an increase in a center wavelength of the signal is desirable for enhancing the penetrating capability of electromagnetic waves through walls. However, an increase in the wavelength is again restricted by two factors: the first one is related with shielding sounding signals by metallic meshes in concrete walls, while the second one decreases the RCS of the target when the wavelength exceeds the sizes of the target. The estimation carried out have shown that for conducting of rescue activities in ruins, typical concrete buildings and facilities the most optimal is the frequency range 0.8 to $2 \mathrm{GHz}$.

\section{Effects of wall and human body as radar target}

\section{A. Wall clutter $[3,4]$}

In through wall target detection, clutter can be due to many reasons like wall coupling, antenna coupling, multiple reflections.

In through wall target detection, clutter reduction can play important part to accurately detect the target and remove the unwanted signals which arise due to the reflection from the wall and other reflections due to unwanted objects. Once the signal is transmitted through the antenna, it suffers attenuation due to wall and other obstacles. A clutter reduction technique such as SVD reduces signal due to wall and enhances the peak due to target.

\section{B. Human target detection [4-6]}

Detection of human beings with radars is based on movement detection (e.g., walking human), chest movements due to breathing or heartbeat. Heart beat and respiratory motions cause changes in frequency, phase, amplitude, and arrival time of reflected signal from a human being. In case of through wall human target detection, these changes can be very small, especially for a brick or concrete walls.

Reflected UWB signal is highly sensitive to human posture and thus makes detection process challenging. For example, the signal reflected from the breathing human causes changes in received waveform shape.

An effective human detection method requires a model of UWB radar waveform propagation and scattering, e.g., interaction with the human body. A perfectly reflecting target e.g. a metal plate with an infinite area returns the incident UWB pulse along a single-path. However, for a target such as human body, which has complex shape and whose spatial extent is larger than the transmitted UWB signal pulse width, the returned UWB radar signal consists of multipath components, as the incident UWB pulse scatters independently from different human body parts at different times with different amplitudes (depending on the distance to the body part and the size, shape, and composition of the scattering part).

\section{Measurements}

This article considers P220 UWB radar in monostatic mode (shown in Figure 1) where waveform pulses are transmitted from a single Omni-directional antenna and the scattered waveforms are received by a collocated Omni-directional antenna [1,7]. The two antenna ports on the P220 are used for the transmit and receive antennas. An Ethernet cable is used to connect the radio to the $\mathrm{PC}$ and radar can be controlled using application software provided with the radios. The P220 UWB radar used here has center frequency of $4.3 \mathrm{GHz}$ with a $10-\mathrm{dB}$ bandwidth of $2.3 \mathrm{GHz}$. This radar provides a resolution of $6.5 \mathrm{~cm}$.

In this section, we look at few important related parameters related to radio configuration. These parameters are important in analyzing captured scans.

Integration is the number of radio pulses that radar combines to increase the signal-to-noise ratio. It is the total number of UWB pulses per waveform (scan) sample.

Window Size $(f t)$ is the width of the 'window', in which motion can be detected.

Pulses Per Waveform is the number of UWB radio pulses required for the entire waveform (single scan). Divide this by the pulse rate to determine the theoretical maximum scan rate.

Step Size ( $p s)$ is the waveform scan resolution (step size between points), in picoseconds ( $1 \mathrm{bin}=3.18 \mathrm{pS}$ ).

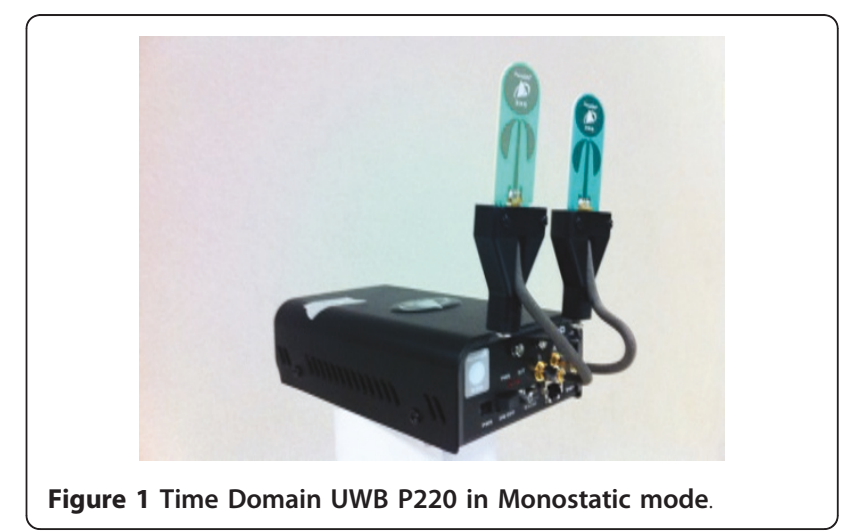




\section{A. Data collection}

For each measurement set, scans were acquired for duration of around $1 \mathrm{~min}$. The number of scans acquired depends on the scan rate which in turn depends on the waveform scan resolution, the window size, and the Integration size. Also scans were taken once with human target and once without human target.

The equations below show the relation between some important parameters.

$$
\text { Number of sample points per scan }=\frac{2 * \text { window size } * 0.3049}{c * \text { step size }(\text { bins })}
$$

Number of pulses per scan $=$ Integration ${ }^{*}$ number of sample points per scan

$$
\text { Scan rate }=\frac{\text { Number of pulses } / \text { scan }}{\text { Pulse repetition frequency }}
$$

Total number of scans collected $=$ scan rate $*$ total data collection time

From the above expressions, we can see that increasing the scan Window Size or Integration size increases the scan time and thus reduces the Scan Rate. However, increasing the Step Size decreases the Scan Rate.

\section{B. Measurement locations}

For the purpose of this project, measurements were taken at four different locations having different types of walls. The radar parameters in each of the cases given below were

Integration: Hardware Integration $=512$, Software Integration $=2$, Pulse Repetition Frequency: $9.6 \mathrm{MHz}$

Step Size: 1 bin, 7 bin, Window Size (ft): $10 \mathrm{ft}$

\section{(1) Gypsum wall}

Figure 2 shows the location of the radar and Human target on different sides of a 1-ft thick Gypsum partition wall. Person is at a distance of $6.5 \mathrm{ft}$ from the radar on the other side of the wall and the height of the antennas from ground is 3'4".

\section{(2) Wooden door}

Figure 3 shows the location of the radar and Human target on different sides of a 4-cm wooden door. Person is standing at a distance of 7'6" from the radar on the other side of the door and the height of the antennas from ground is 3 '4 ".

\section{(3) Brick wall}

Figure 4 shows the location of the radar and Human target on different sides of a 12-cm Brick wall. Person is standing at a distance of 8 ' from the radar on the other side of the door and the height of the antennas from ground is 3'4".

\section{(4) Load bearing concrete wall}

In this case, measurements were taken at two different positions as shown in Figure 5. In both cases, person is standing at 7'6" from the radar and the height of the radar is $3{ }^{\prime} 4$ ".

\section{Measurement analysis}

In this section, we discuss the three approaches that are used in this article to analyze the measurements.

\section{A. Detection of breathing movements}

This approach is based on detection of small chest movements associated with a breathing motionless human. This motion is very small and results in very weak radar echo. However, since it is periodic motion it can be detected by application of signal processing techniques which enhances the 'breathing' signal from noise.

Breathing motion will cause periodic changes in the received signal at a distance where target is located. This periodic change is reflected across multiple scans. Thus an $N \times M$ matrix $\mathbf{A}$ is constructed using ' $\mathrm{M}$ ' scans, each of length ' $N$ ', as columns of matrix $\mathbf{A}$. Then difference is taken between successive columns of matrix A, which captures changes from one scan to another and helps to suppress the static clutter signal.
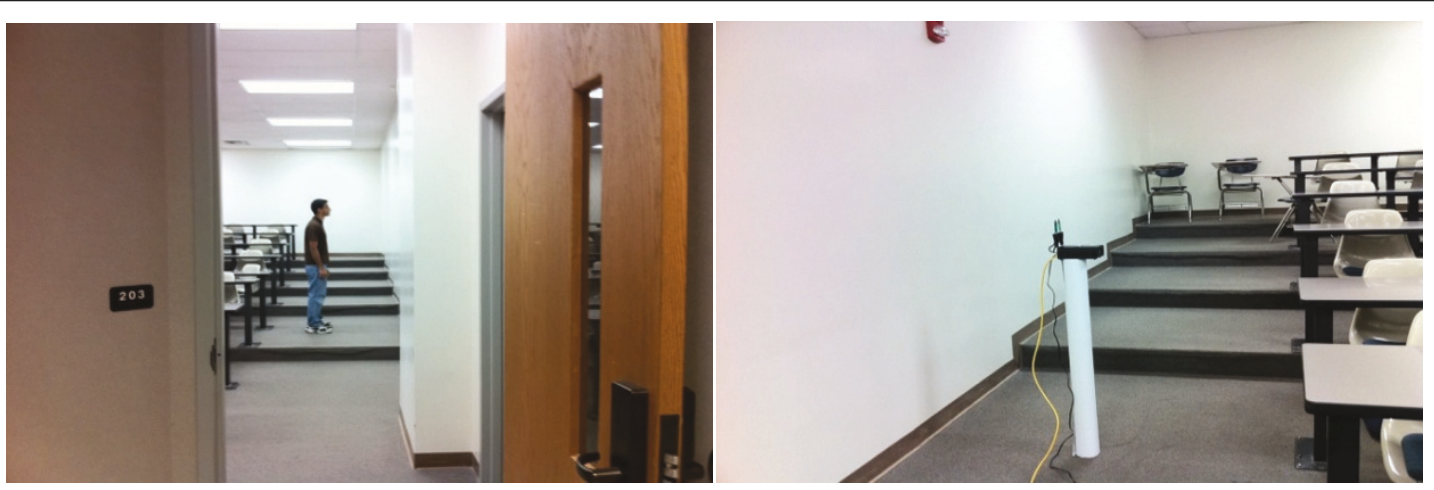

Figure 2 UWB radar (Right), Human target (Left) for gypsum wall. 


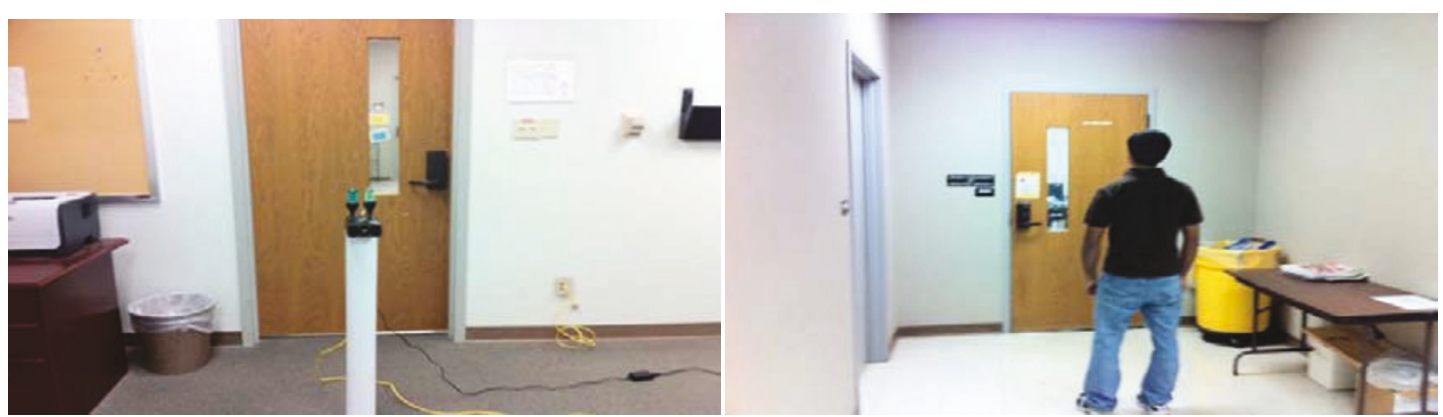

Figure 3 UWB radar (Left), Human target (Right) for wooden door.

Finally DFT is performed on each row of the resulting matrix which clearly shows the breathing human target. This approach is summarized below.

Step 1. Matrix A constructed using ' $M$ ' scans arranged in columns

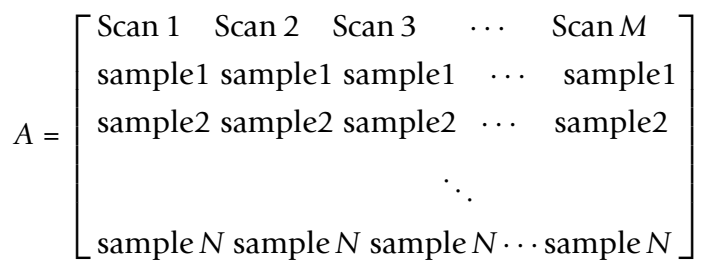

Step 2. Matrix D is the difference between successive columns of $\mathbf{A}$

$$
D=\left[\begin{array}{lll}
\text { Scan1 }- \text { Scan2 } & \text { Scan2 }- \text { Scan3 } & \text { Scan }(M-1)-\text { ScanM } \\
\text { sample1 } & \text { sample1 } & \text { sample1 } \\
\text { sample2 } & \text { sample2 } & \text { sample2 } \\
\vdots & \vdots & \vdots \\
\text { sampleN } & \text { sampleN } & \text { sampleN }
\end{array}\right]
$$

Step 3. Take Discrete Fourier Transform of each row of the Matrix $\mathbf{D}$.

This technique works for gypsum wall, wooden door, and brick wall. Below are the observations for these cases.

\section{(1) Gypsum wall}

Figure 6 shows the D-Matrix with and without target. In this case, D matrix is constructed using 100 scans captured at scan rate of 0.6827 scans/s for total time duration of $68 \mathrm{~s}$.

A discrete Fourier transform (DFT) on each row of Dmatrix shows the breathing rate of a human target at 6.5 ft. (Figure 7).

Figure 8 shows the case where the person is moving his hands towards the radar and back at rate close to 1 $\mathrm{Hz}$

\section{(2) Wooden door}

See Figures 9 and 10.

\section{(3) Brick wall}

See Figure 11.

This method of detecting motionless people may not work in all cases. For example, this approach works well for wooden door, gypsum partition wall, and brick wall as shown above but fails when the attenuation for signal scattered from target is large compared to the signal reflected from wall or other stationary objects (e.g., concrete wall). In such cases, detection of weak target signal in presence of strong clutter from wall is difficult and will require use of some kind of clutter reduction method. Also this method may fail when the person has his back towards the wall as the chest movements may not be captured in the resulting scans.

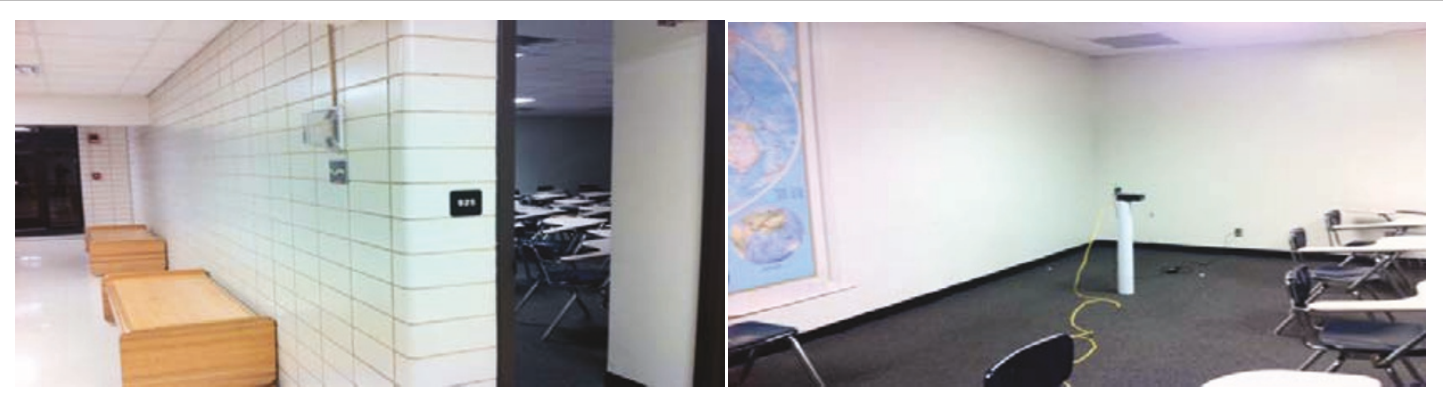

Figure 4 UWB radar (Right), Human target position close to bench farther away in image (Left) for brick wall. 

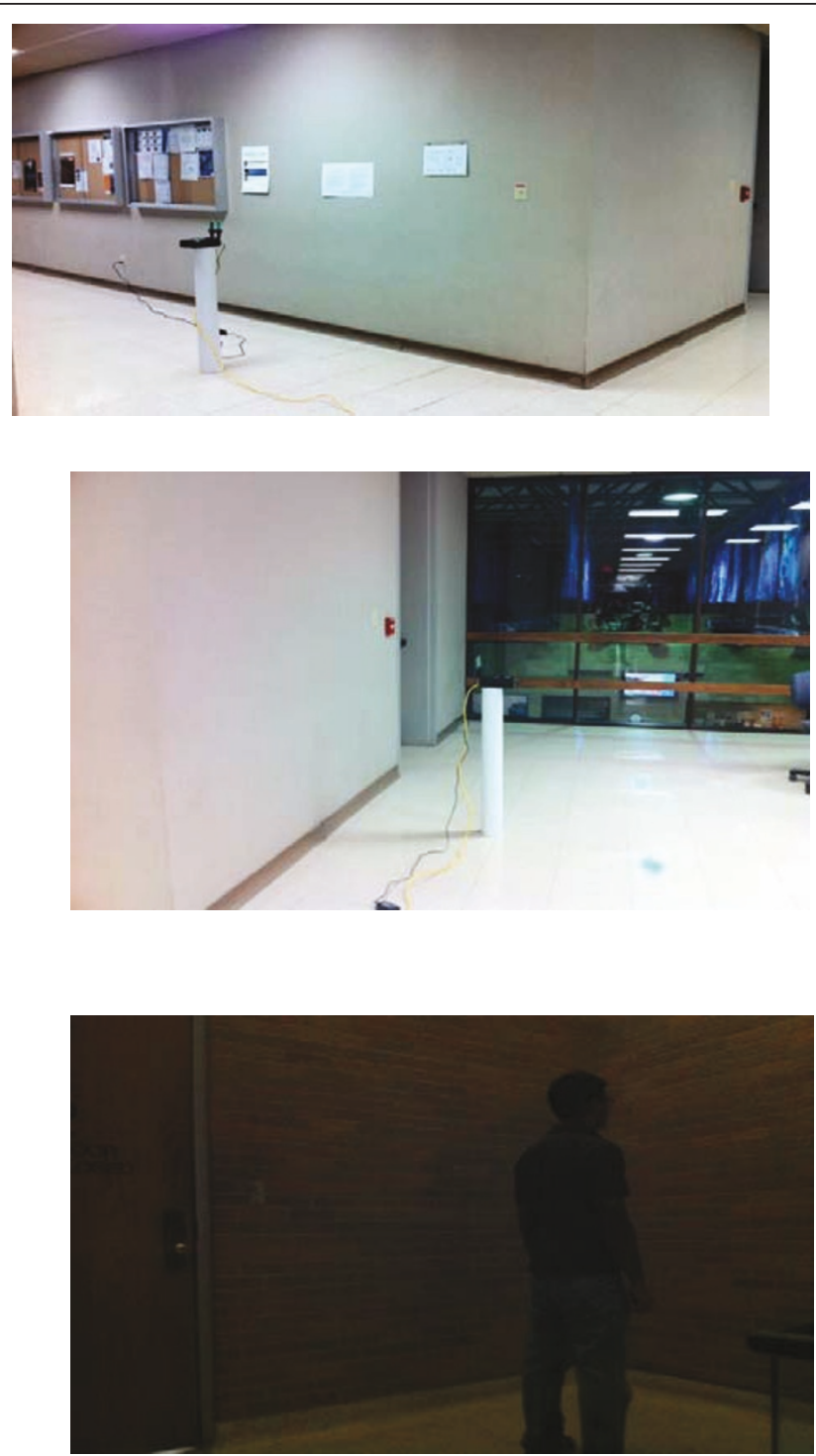

Figure 5 UWB radar position 1(Top), UWB radar position2 (Middle), Human standing behind Concrete wall (Bottom) for brick wall.

\section{B. Clutter reduction using SVD $[8,9]$}

SVD is used here to reduce wall clutter. The main aim of SVD is to split the Scan-Matrix into subspaces which correspond to clutter, target, and noise so that the clutter can then be rejected. The Scan-Matrix is constructed by arranging ' $M$ ' scans each of length ' $N$ ' in matrix format giving an $N \times M$ Matrix A. Each column of this matrix is a single scan of length $M$. The SVD of A is given as

$$
\mathrm{A}=\mathrm{USV}^{\mathrm{T}}
$$

where $\mathbf{U}^{\mathbf{T}} \mathbf{U}=\mathbf{I} ; \mathbf{V}^{\mathbf{T}} \mathbf{V}=\mathbf{I}$; the columns of $\mathbf{U}$ are orthonormal eigenvectors of $\mathbf{A A}^{\mathbf{T}}$, the columns of $\mathbf{V}$ are orthonormal eigenvectors of $\mathbf{A}^{\mathbf{T}} \mathbf{A}$, and $\mathbf{S}$ is a diagonal matrix containing the square roots of eigenvalues from
$\mathbf{U}$ or $\mathbf{V}$ in descending order.

$$
\begin{aligned}
& \mathrm{A}=\sigma_{1} u_{1} v_{1}^{T}+\sigma_{2} u_{2} v_{2}^{\mathrm{T}}+\sigma_{3} u_{3} v_{3}^{\mathrm{T}}+\ldots \\
& \mathrm{A}=\mathrm{M}_{1}+\mathrm{M}_{2}+\mathrm{M}_{3}+\ldots
\end{aligned}
$$

where, $\mathbf{M}_{i}$ is called as the $i$ th Eigen-image of $\mathbf{A}$

It has been found experimentally that first Eigenimage corresponds to clutter, second Eigen-image corresponds to target and the rest are noise [2]. Therefore, we have

$$
A=M_{\text {clutter }}+M_{\text {target }}+M_{\text {noise }}
$$

where, $\mathrm{M}_{\text {clutter }}=\sigma_{1} u_{1} v_{1}^{\mathrm{T}}, \mathrm{M}_{\text {target }}=\sigma_{2} u_{2} v_{2}^{\mathrm{T}}$, and $\mathrm{M}_{\text {noise }}=\sigma_{3} u_{3} v_{3}^{\mathrm{T}}+\sigma_{4} u_{4} v_{4}^{\mathrm{T}}+$. 


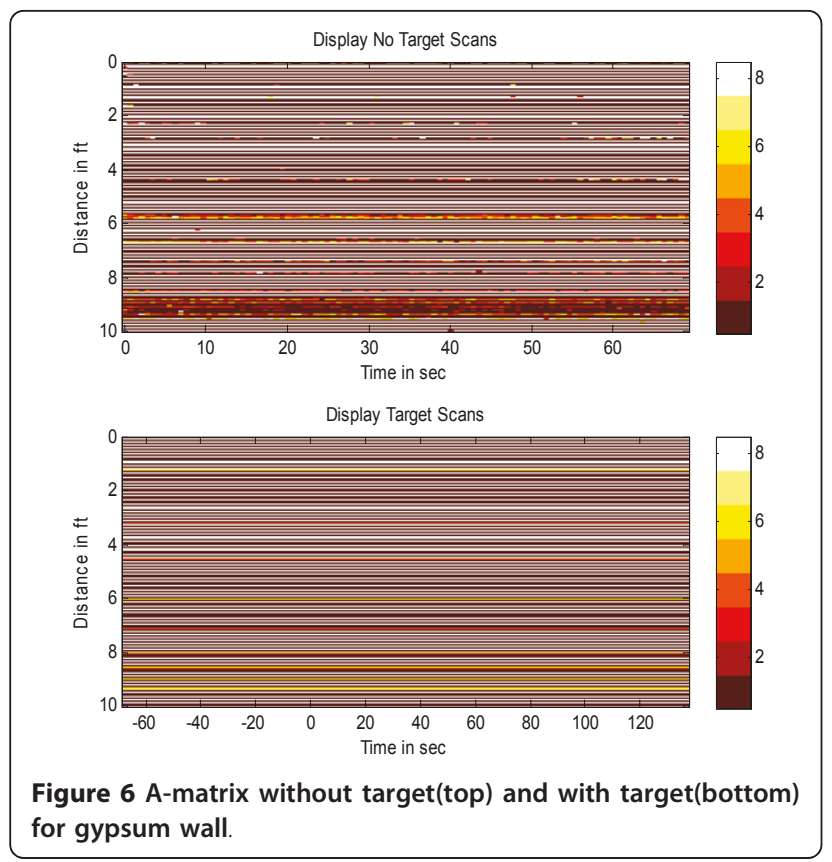

This technique does not work for the case of concrete wall and wooden door.

\section{(1) Gypsum wall}

Here after applying SVD to A-Matrix, clutter is reduced and target can be detected. Figures show the A-matrix with target (Figure 12), Eigen Image corresponding to clutter (Figure 13) and target (Figure 14).

\section{(2) Brick wall}

See Figures 15, 16 and 17.

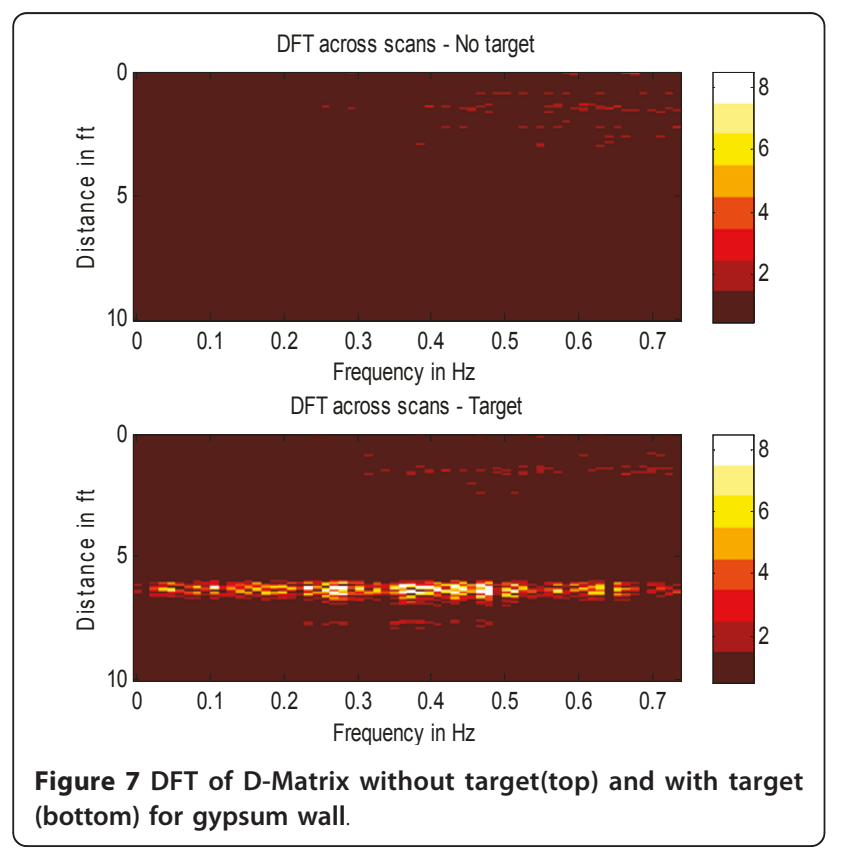

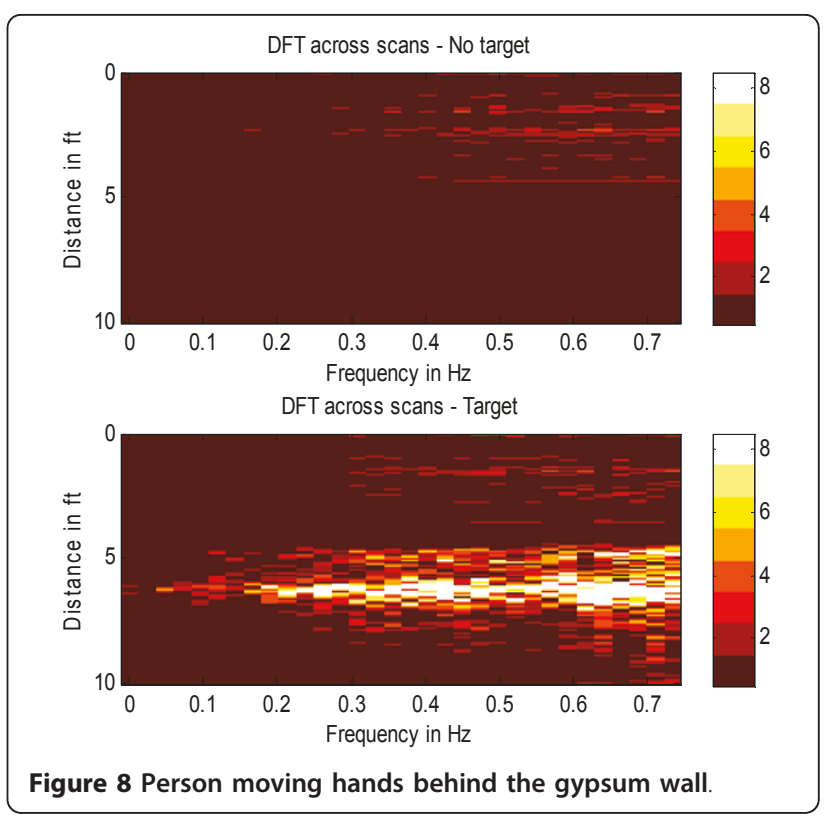

\section{Short time Fourier transform and singular value decomposition}

Short time Fourier transform (STFT) is a tool to analyze frequency contents of signals that vary in time. STFT maps a signal into a two-dimensional function of time and frequency. It represents a kind of compromised view of signal in time and frequency. However, this information is obtained with limited precision and this precision is determined by the window size.

Analysis using STFT involves choosing appropriate window size so as to get good resolution in both time and frequency domain as there is always a trade-off between the two. The window type is selected according to compromise between sidelobe attenuation

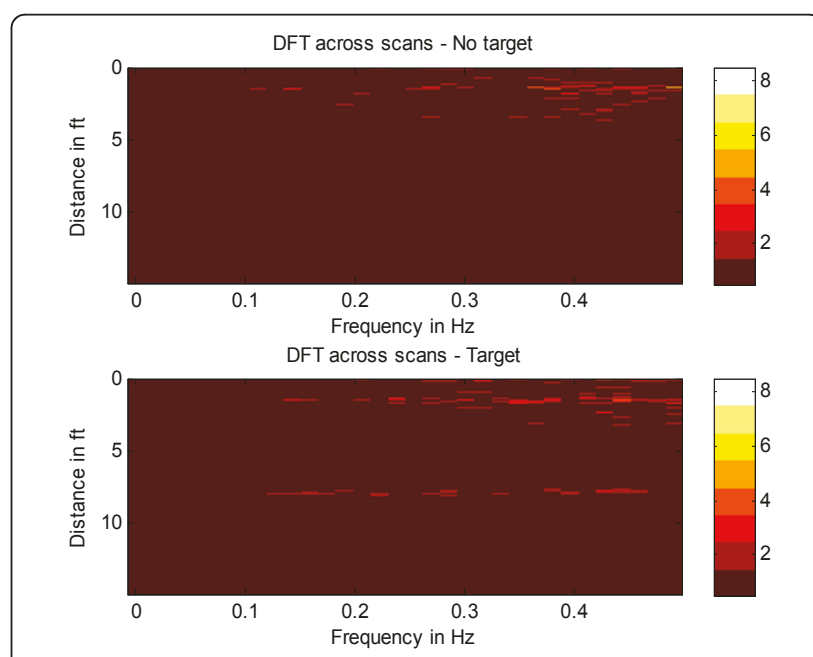

Figure 9 DFT of D-Matrix without target (top) and with target (bottom) for wooden door 


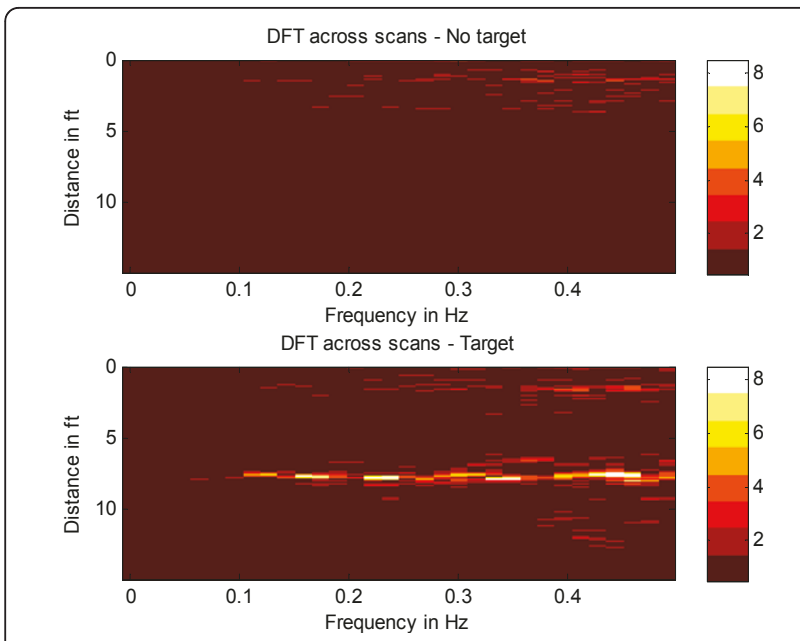

Figure 10 Person moving hands behind the wooden door

requirements and mainlobe width (usually Hanning or Blackman are used). Window size needs to be adapted to the signal and the information one is looking for.

STFT of a single scan will provide information content about frequencies across the scan duration. Then SVD is done on the STFT output to see if the target can be identified based on its frequency content.

However, selecting an STFT window size that will result in enough resolution in both time and frequency to identify the target and its distance is quiet challenging.

Various window sizes were tried for STFT to see if there is any difference in the singular values obtained from the SVD for target and no target case.

\section{(1) Gypsum wall}

Figure 18 shows the STFT for the no target scan and Figure 19 shows STFT for target case. Window size

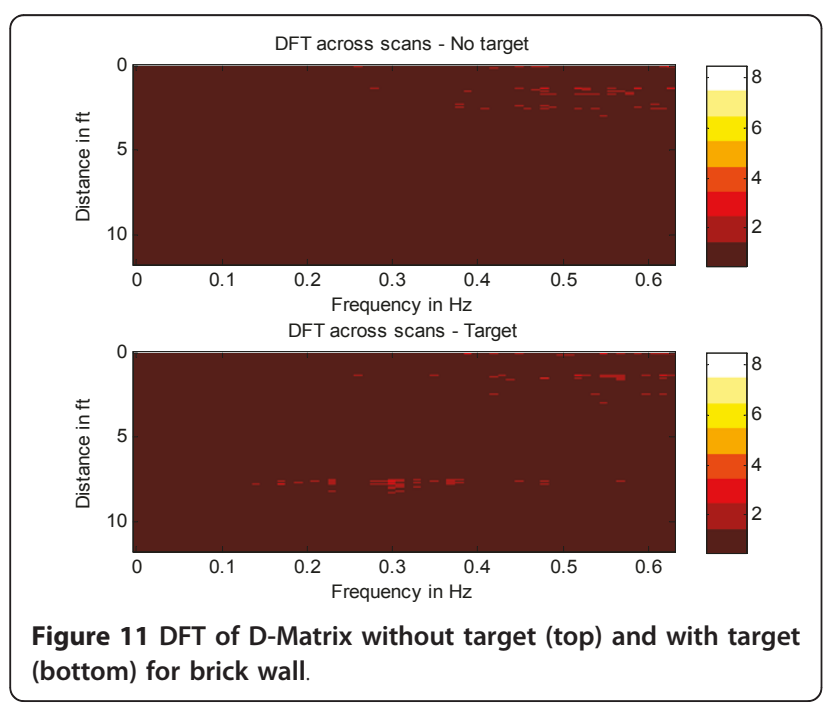

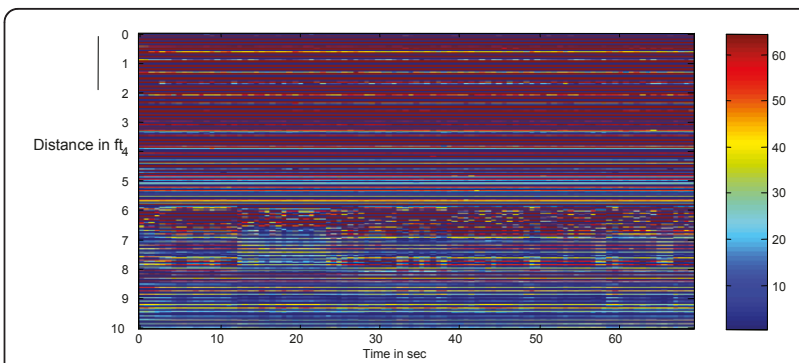

Figure 12 A-matrix with target for gypsum wall.

used in this case is 512 with an overlap of 128 . Target is located around 4155 sample index.

The singular values obtained from the STFT data are normalized by dividing each value with the maximum singular value, as plotted in Figures 20 and 21. It is observed in that there is relative increase in the second singular value in case when target is present. This relative increase is around 0.2. However, this is not consistent when applied to other cases of wooden door, brick wall, and concrete wall.

\section{Conclusion and future work}

For detection of human target using UWB radar, various sets of measurements were taken using monostatic radar mode. Data were collected for different types of walls and doors. The scans collected were analyzed using three different approaches. It is observed that the heart beat detection using Doppler approach works for wooden door, gypsum, and brick wall but fails in case of a thick concrete wall. A second method using singular value decomposition was used to reduce clutter and this works for brick and gypsum wall but again fails for concrete wall case. Finally, we tried an STFT and SVD method based on the idea that the received signal in case of presence of target will result in difference in frequency response compared to no target case. In this method, selection of window size and overlap size is a challenging task. By applying SVD to the STFT output it is observed, in case of gypsum wall, that the second singular value changes relatively in presence of target.

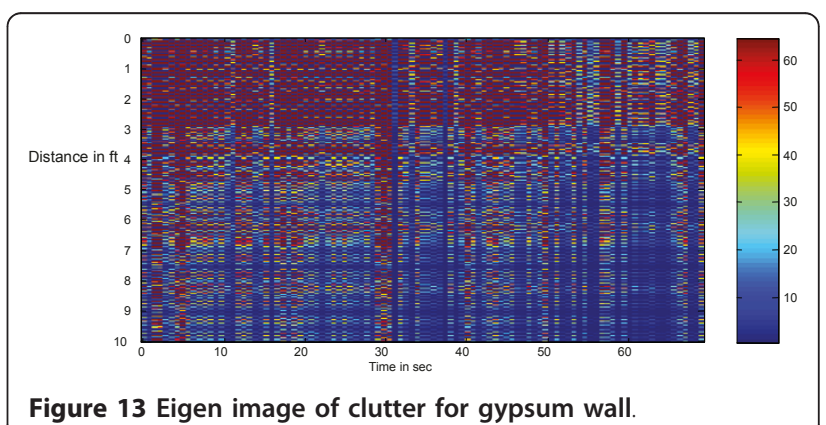




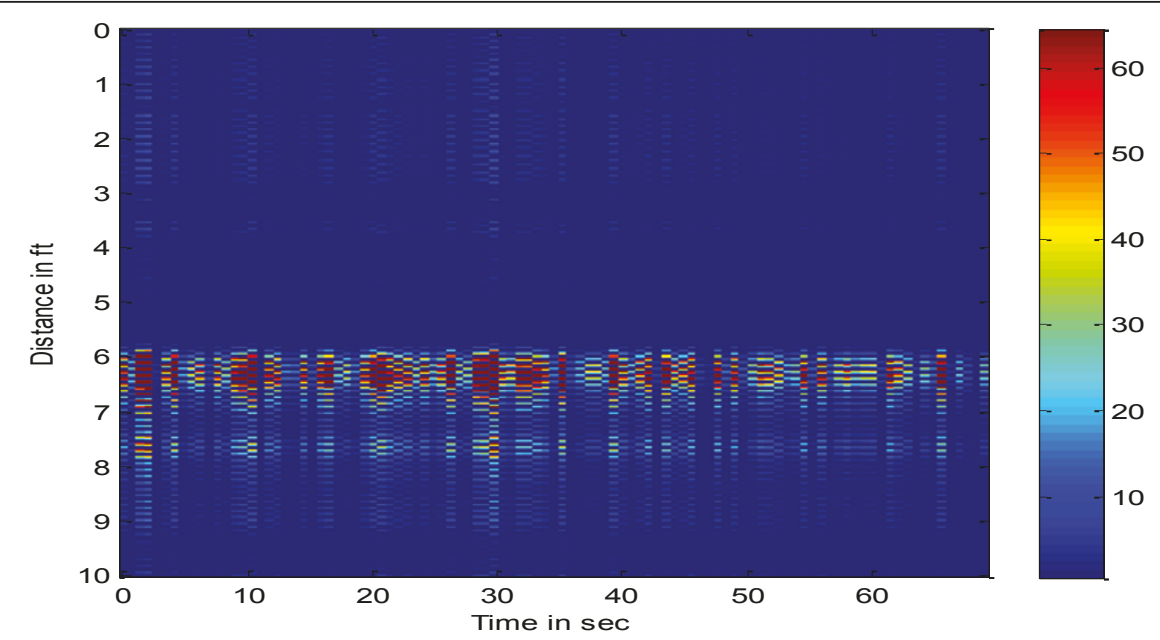

Figure 14 Eigen image of target for gypsum wall.

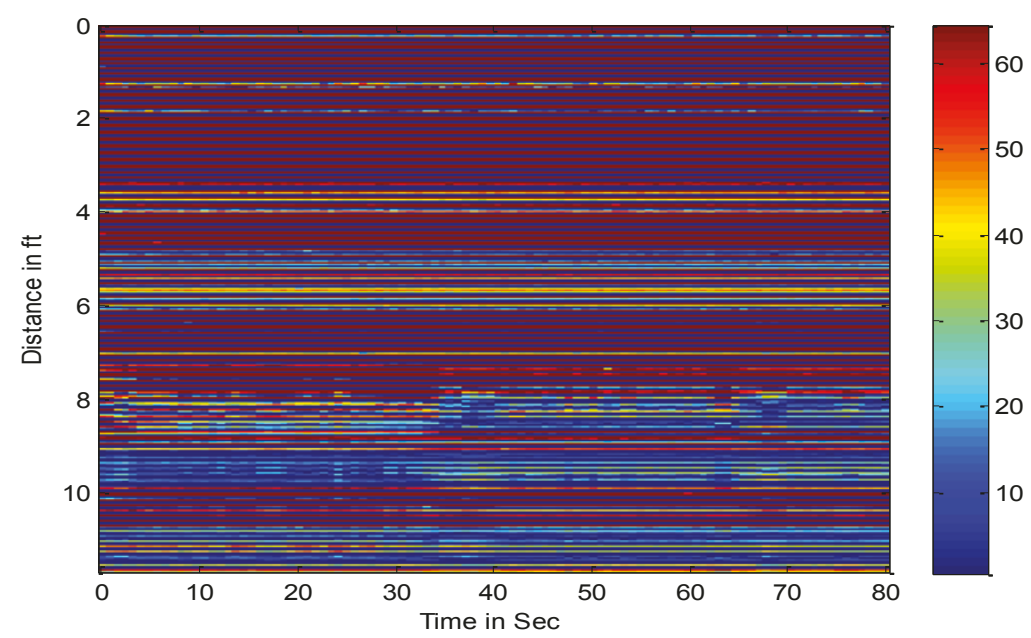

Figure 15 A-matrix with target for brick wall.

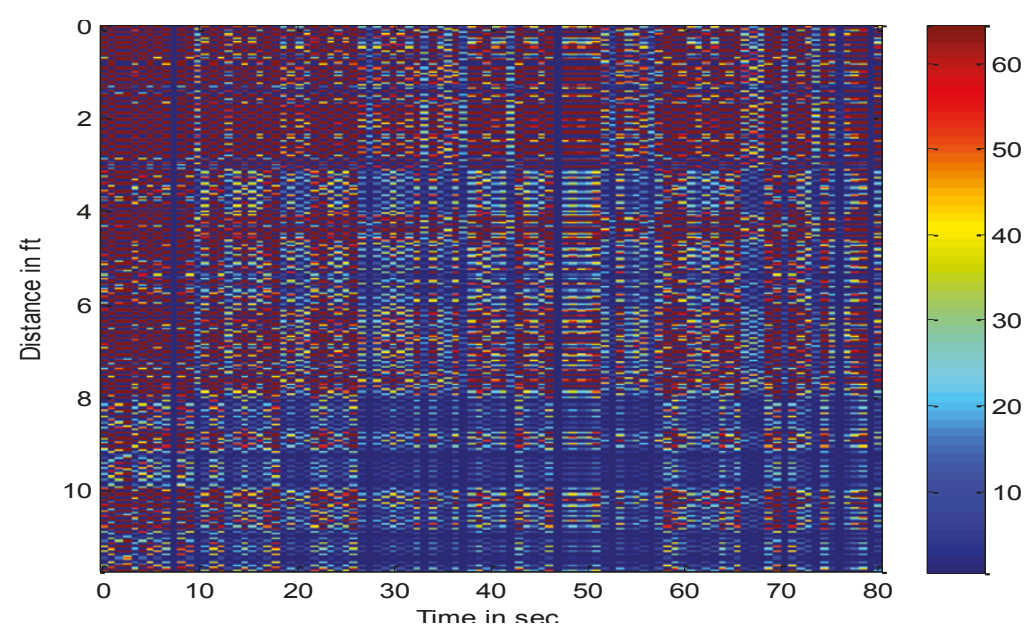

Figure 16 Eigen image of clutter for brick wall. 


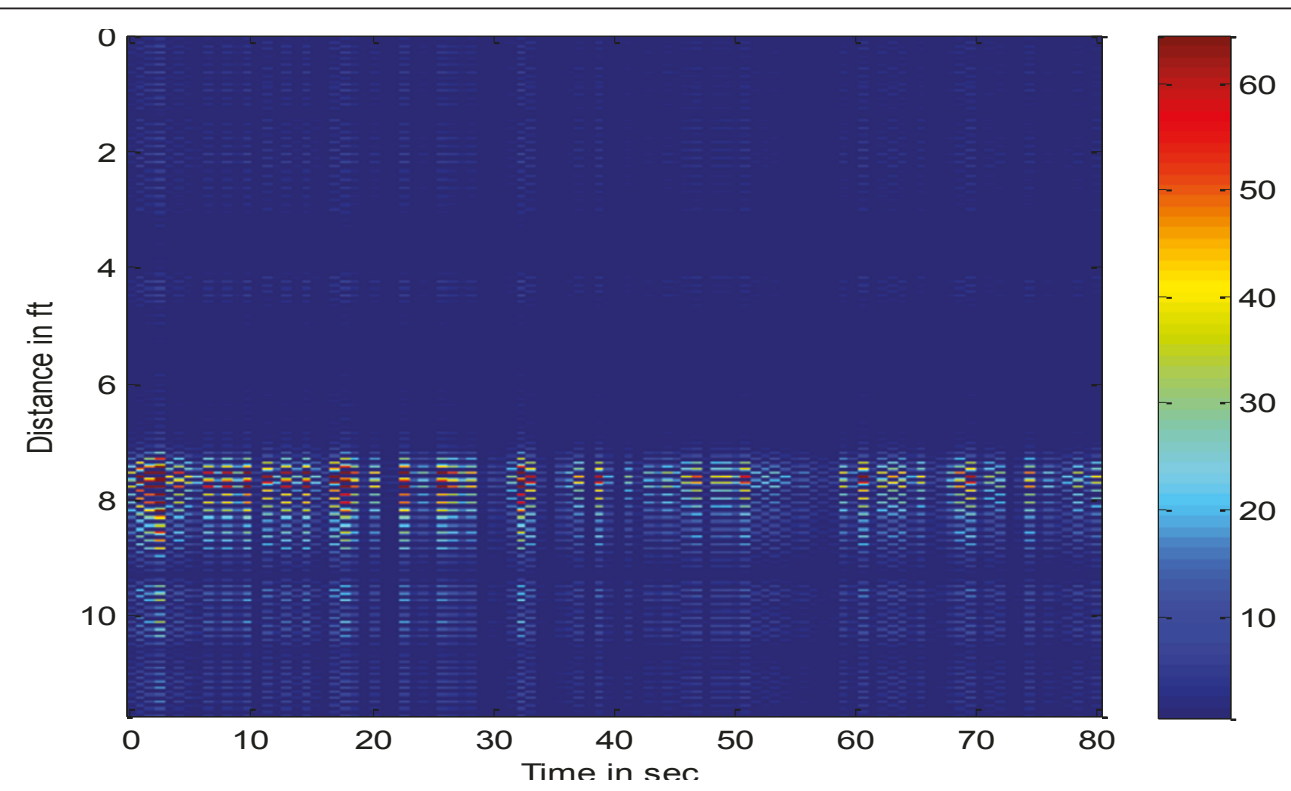

Figure 17 Eigen image of target for brick wall.

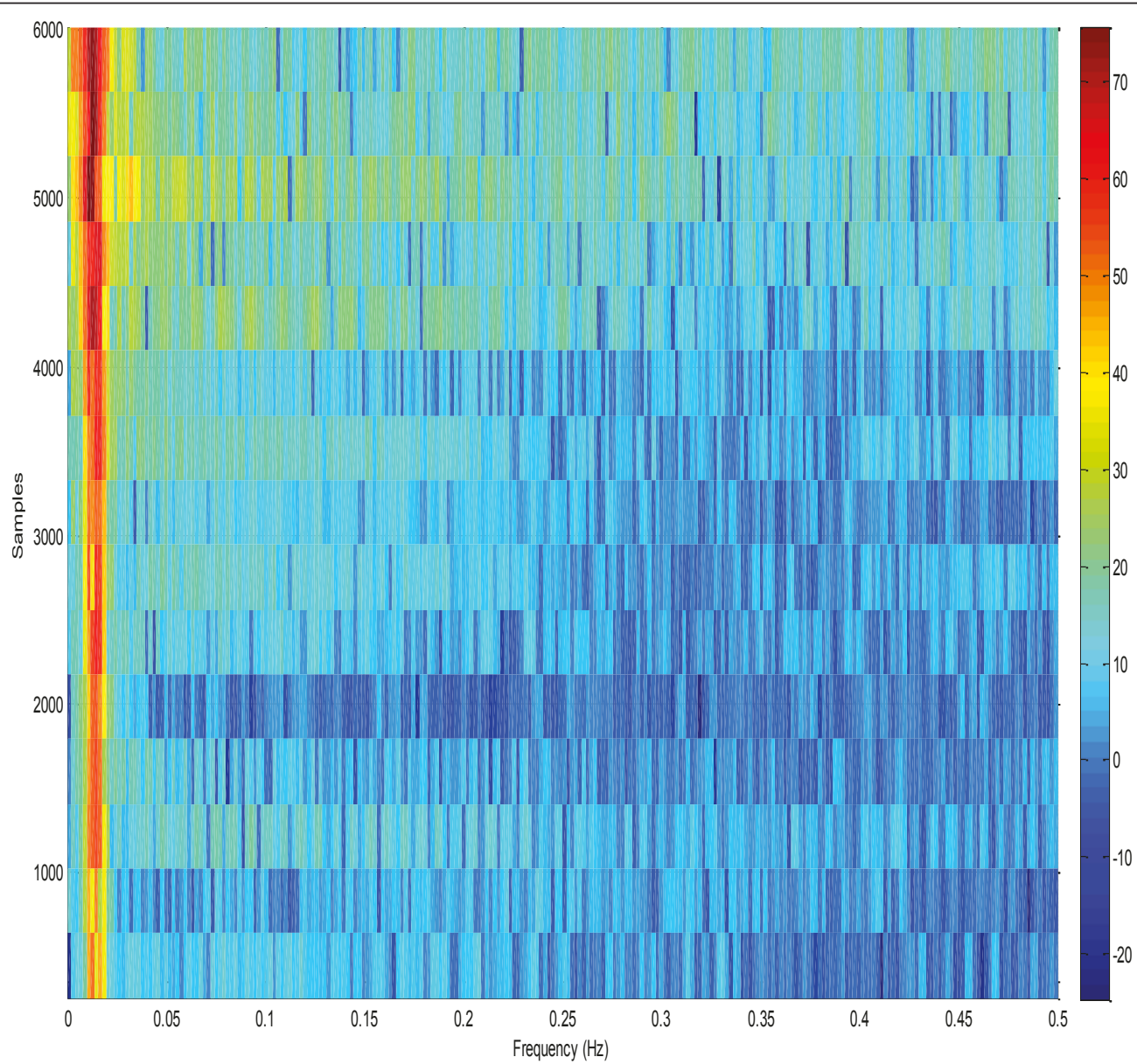

Figure 18 STFT of single scan with no target for gypsum wall. 


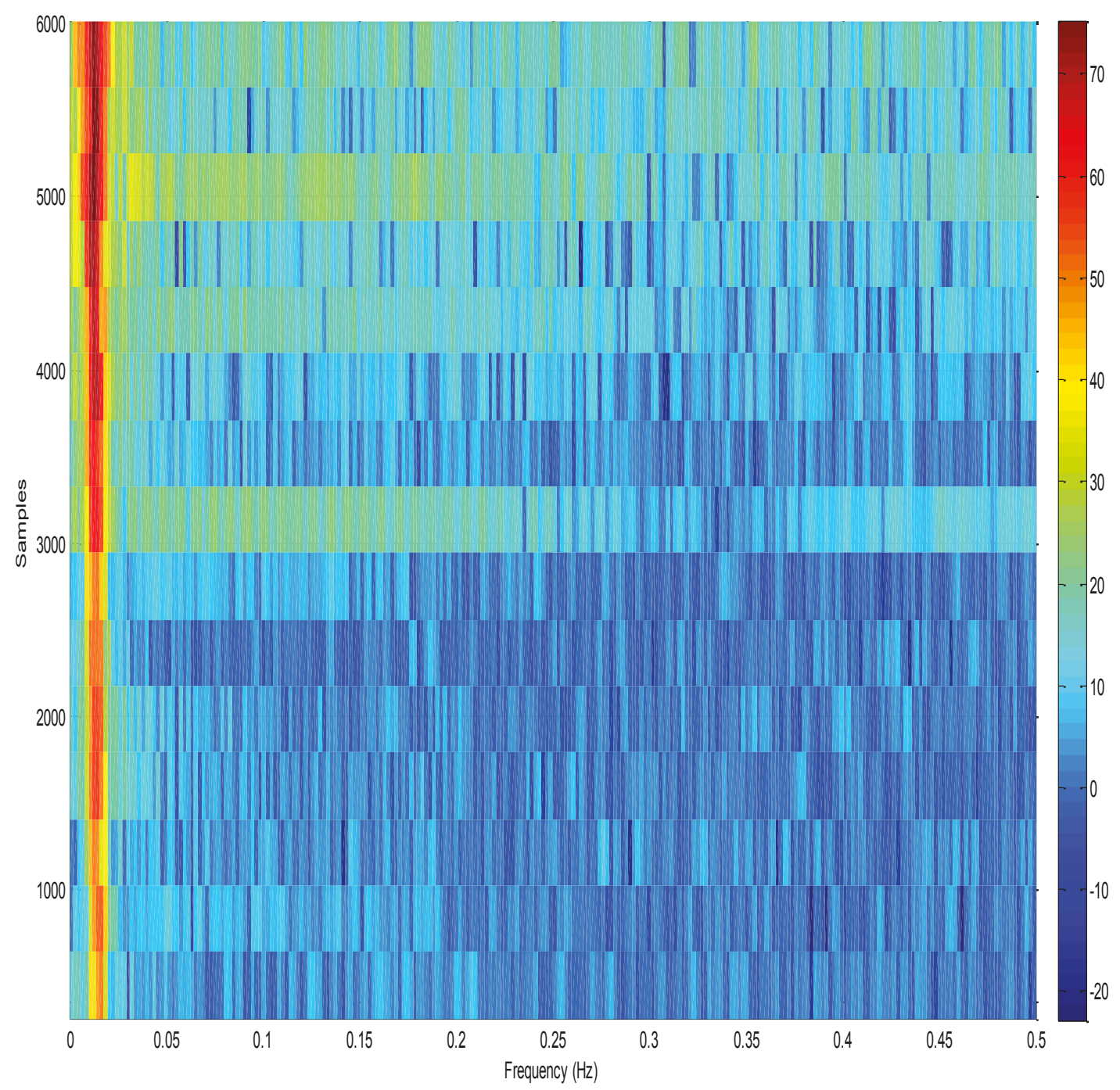

Figure 19 STFT of single scan with target for gypsum wall.
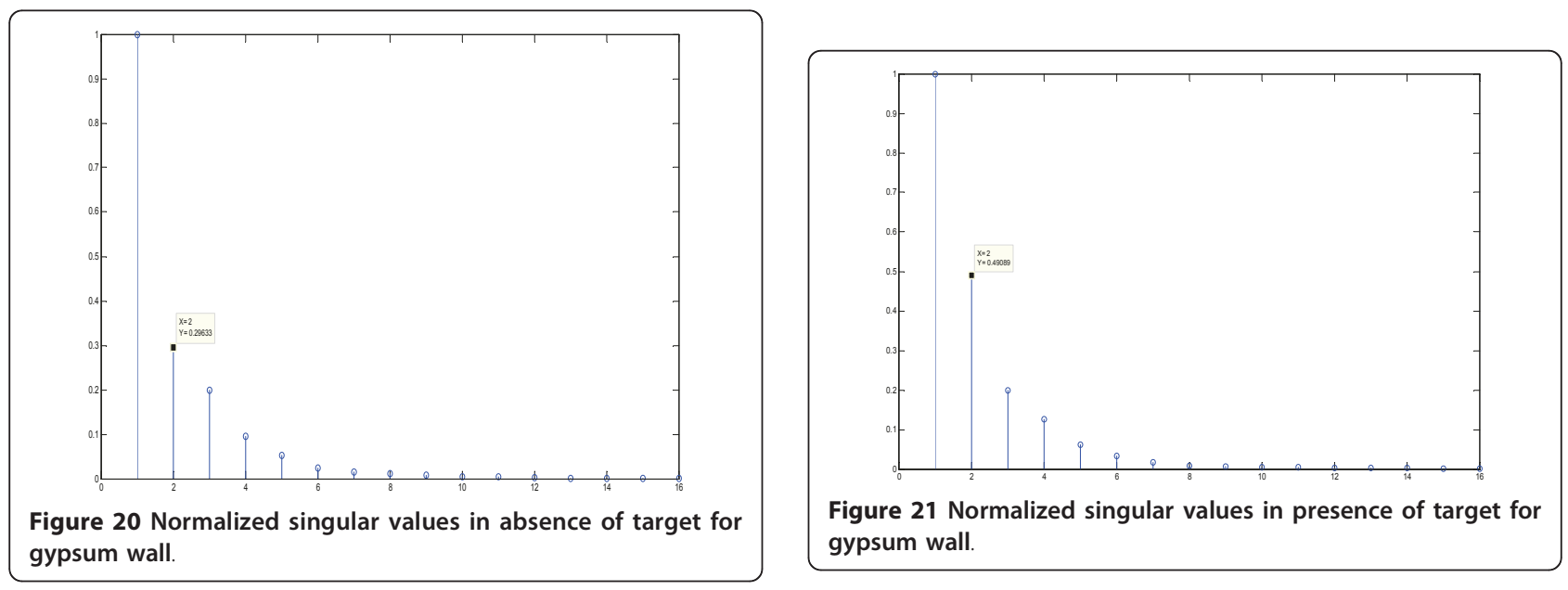
However, the approach does not work for other cases. The breathing rate detection approach does work for all except the concrete wall case, but cannot be entirely relied upon as the detection process is based on changes in the received signal amplitude. For example, the received signal amplitude may be very weak for person standing with his/her back to the wall under consideration.

Given the drawbacks of different approaches used in this project, a new approach is required which should work for all cases. Also while collecting data, only monostatic mode of operation was considered. Therefore, a Bi-static operation mode could be considered and also multiple locations along the wall can be considered to perform some kind of spatial filtering to reduce the wall clutter.

\section{Abbreviations}

DFT: discrete Fourier transform; SVD: singular value decomposition; STFT: short time Fourier transform; UWB: Ultra Wide Band.

\section{Acknowledgments}

This work was supported in part by Office of Naval Research (ONR) under grant N00014-11-1-0071 and National Science Foundation (NSF) under grant CNS-1050618, CNS-0964713, and CNS-0964060.

\section{Author details}

'Department of Electrical Engineering, University of Texas at Arlington, Arlington, TX, 76019-0016, USA ${ }^{2}$ Department of Preventive Medicine and Biometrics, Uniformed Services University of the Health Sciences, Bethesda, MD, 20814-4799, USA ${ }^{3}$ Department of Mathematics, Drexel University,

Philadelphia, PA, 19104, USA

\section{Competing interests}

The authors declare that they have no competing interests.

Received: 15 February 2011 Accepted: 21 June 2011

Published: 21 June 2011

\section{References}

1. LE Miller, Why UWB? A review of Ultrawideband Technology. National Institue of Standards and Technology, Report to NETEX Project Office, DARPA, http://www.antd.nist.gov/wctg/manet/NIST_UWB_Report_April03. pdf (2003)

2. Qilian Liang, Biologically-Inspired Target Recognition in Radar Sensor Networks, EURASIP Journal on Wireless Communications and Networking, Paper ID: 523435, vol. 2010

3. K Lukin, V Konovalov, Through wall detection and recognition of human beings using noise radar sensors. Proc NATO RTO SET Symposium on Target Identification and Recognition Using RF Systems, Oslo, Norway, P15-1-P15-11, October 2004

4. J Liang, Signal processing in radar and non-radar sensor networks. PhD Dissertation, The University of Texas at Arlington http://dspace.uta.edu/ handle/10106/1727 Auquest 2009

5. Stephen Crabbe, Michal Aftanas, Per-Anders Berthlin, Miloš Drutarovský, Ralf Klukas, prof. Dušan Kocur, Trung Thanh Nguyen, Dr. Peter Peyerl, Jana Rovnáková, Dr. Jürgen Sachs, Egor Zaikov, Ultra wideband radar for through wall detection from the RADIOTECT project. Fraunhofer Symposium, Future Security, 3rd Security Research Conference Karlsruhe, (ISBN 978-3-81677598-0) 299

6. AG Yarovoy, LP Ligthart, J Matuzas, B Levitas, UWB radar for human being detection. IEEE Aerospace and Electronic Systems Magazine. 21(3), 10-14 (Mar. 2006). doi:10.1109/MAES.2006.1624185
7. The Gaussian Monocycle Pulses, Fractional Derivatives, UWB Wireless, and the FCC Part 15 SpectralMasks, Technical Report NDT17-09-2007, 5 September 2007, NanoDotTek (18 pages)

8. R Chandra, AN Gaikwad, D Singh, MJ Nigam, An approach to remove the clutter and detect the target for ultra-wideband through-wall imaging. Journal of Geophysics and Engineering. 5, 412-419 (2008). doi:10.1088/ 1742-2132/5/4/005

9. PK Verma, AN Gaikwad, D Singh, MJ Nigam, Analysis of clutter reduction techniques for through wall imaging in UWB range, Progress In Electromagnetics Research B.17, 29-48

doi:10.1186/1687-1499-2011-20

Cite this article as: Singh et al:: Sense through wall human detection using UWB radar. EURASIP Journal on Wireless Communications and Networking 2011 2011:20.

\section{Submit your manuscript to a SpringerOpen ${ }^{\circ}$ journal and benefit from:}

- Convenient online submission

- Rigorous peer review

- Immediate publication on acceptance

- Open access: articles freely available online

- High visibility within the field

- Retaining the copyright to your article

Submit your next manuscript at $\gg$ springeropen.com 